\title{
Computational drug discovery for the Zika virus
}

\author{
Melina Mottin ${ }^{1}$, Joyce Villa Verde Bastos Borba ${ }^{1}$, Cleber Camilo Melo-Filho ${ }^{1}$, Bruno Junior \\ Neves $^{1,2}$, Eugene Muratov ${ }^{3,4}$, Pedro Henrique Monteiro Torres ${ }^{5}$, Rodolpho Campos Braga ${ }^{1}$, \\ Alexander Perryman ${ }^{6}$, Sean Ekins ${ }^{7}$, Carolina Horta Andrade ${ }^{1,8 *}$
}

\begin{abstract}
${ }^{1}$ Laboratory for Molecular Modeling and Drug Design - LabMol, Faculty of Pharmacy, Federal University of Goiás, Goiânia, GO, Brazil, ${ }^{2}$ Laboratory of Cheminformatics, University Center of Anápolis/UniEVANGÉLICA, Anápolis, GO, Brazil, ${ }^{3}$ Laboratory for Molecular Modeling, Division of Chemical Biology and Medicinal Chemistry, Eshelman School of Pharmacy, University of North Carolina, Chapel Hill, NC, USA, ${ }^{4}$ Department of Chemical Technology, Odessa National Polytechnic University, Odessa, Ukraine, ${ }^{5}$ Instituto Oswaldo Cruz, Fundação Oswaldo Cruz, Rio de Janeiro, RJ, Brazil, ${ }^{6}$ Department of Pharmacology, Physiology and Neuroscience, Rutgers University-New Jersey Medical School, Newark, NJ, United States of America, ${ }^{7}$ Collaborations Pharmaceuticals, Inc., 840 Main Campus Drive, Lab 3510, Raleigh, NC, United States of America, ${ }^{8}$ Laboratory of Tropical Diseases, Department of Genetics, Evolution, Microbiology and Immunology, Institute of Biology, UNICAMP, Campinas, Brazil
\end{abstract}

\begin{abstract}
Few Zika virus (ZIKV) outbreaks had been reported since its first detection in 1947, until the recent epidemics occurred in South America (2014/2015) and expeditiously became a global public health emergency. This arbovirus reached 0.5-1.3 million cases of ZIKV infection in Brazil in 2015 and rapidly spread in new geographic areas such as the Americas. Despite the mild symptoms of the Zika fever, the major concern is related to the related severe neurological disorders, especially microcephaly in newborns. Advances in ZIKV drug discovery have been made recently and constitute promising approaches to ZIKV treatment. In this review, we summarize current computational drug discovery efforts and their applicability to discovery of anti-ZIKV drugs. Lastly, we present successful examples of the use of computational approaches to ZIKV drug discovery.
\end{abstract}

Keywords: Zika. Flavivirus. Antiviral. Computer-assisted drug design. Virtual screening. Drug discovery.

\section{INTRODUCTION}

Zika virus (ZIKV) is an arbovirus that belongs to genus Flavivirus, the same genus of Dengue virus (DENV), Yellow Fever Virus (YFV) and West Nile Virus (WNV) (Wahid et al., 2016). It was first isolated from rhesus monkeys in Uganda, Africa, in 1947 (Dick et al., 1952). After the virus isolation, the disease did not call much attention because only a limited number of human infections were reported. The scenario changed after an outbreak in Micronesia in 2007 and epidemics in other areas across the Pacific (Duffy et al., 2009). In 2014 and 2015 the virus spread increased dramatically in Americas, mostly in Brazil with an estimation of 0.5-1.3 million cases of ZIKV infection at the end of 2015 (Hennessey, Fischer,

\footnotetext{
*Correspondence: C. H. Andrade. Laboratório de Modelagem Molecular e Desenho de Fármacos. Faculdade de Farmácia, Universidade Federal de Goiás, Goiânia, GO, Brazil. E-mail: carolhandrade@gmail.com
}

Staples, 2016). In 2016 the World Health Organization (WHO) declared the ZIKV as a global health emergency due to its quick dissemination and the connection with neuronal diseases (Gulland, 2016).

The typical symptoms of ZIKV disease are not very concerning like fever, joint pain, maculopapular rash, and conjunctivitis. However, the association of the disease with congenital microcephaly (Araújo et al., 2016), Guillain-Barre syndrome (Oehler et al., 2014), and macular atrophy (Ventura et al., 2016) are the concerning aspects of the disease.

Currently, there are no specific vaccines or antiviral drugs available for the disease and many efforts have been made towards the development of new and effective antiviral compounds. Computational approaches have been used for ZIKV drug discovery with successful results. Besides the acceleration of the discovery process, these techniques reduce experimental costs, decrease the use of animals in experiments and are environment-friendly. 
Here we review the computational techniques applied for the discovery of compounds that might stop ZIKV spreading.

\section{COMPUTATIONAL TECHNIQUES APPLIED TO ZIKV DRUG DISCOVERY}

The structural and biological knowledge about ZIKV is indisputably important for successfully guiding drug discovery projects. However, the infrastructure requirements for these experimental assays are beyond the reach of most researchers due to its high cost. To make experimental assays less expensive, researchers can use computer-assisted drug discovery (CADD) as a complementary approach to prioritize compounds for synthesis and/or biological evaluation. As no compound need be tested before computational simulations, CADD represents a time-, labor-, and cost-effective strategy to obtain antiviral compounds in the early stages of ZIKV drug discovery projects (Ekins et al., 2016b; Joy Macalino et al., 2015; Kar, Roy, 2013; Reynolds, 2014).

Typically, CADD approaches are applied in virtual screening (VS) campaigns. VS is often compared to a funnel, where thousands/millions of chemical structures (i.e., $10^{5}$ to $10^{7}$ compounds) available on virtual libraries are filtered by a computational model to a smaller set of potentially active chemicals (i.e., $10^{1}$ to $10^{3}$ compounds) that will then be synthesized and/or tested experimentally (Kar, Roy, 2013; Tanrikulu, Krüger, Proschak, 2013). Compared with typical hit rates from experimental approaches that can range between $0.01 \%$ and $0.14 \%$, VS typically range between $1 \%$ and $40 \%$ (Zhu et al., 2013). Because of their obvious advantages, CADD approaches are widely employed in academic organizations and pharmaceutical industry. In the subsequent sections, we review current developments in CADD, highlighting main strategies and pitfalls that have implications in ZIKV drug discovery projects.

\section{Ligand-based drug design (LBDD)}

Usually, when the biological target is not known or its 3D structure is not available, CADD can use the chemical structures of known active compounds (i.e., obtained from biochemical or phenotypic assays) as starting points. This approach is known as Ligand-based drug design or LBDD (Glaab, 2016; Ripphausen, Nisius, Bajorath, 2011). There are four main LBDD methods: (i) similarity search; (ii) 3D shape matching; (iii) ligandbased pharmacophores; and (iv) quantitative-structure activity relationships (QSAR).
Similarity search

Ligand similarity search is based on the principle that structurally similar compounds exhibit similar biological activities. Figure 1A shows the schematic representation of calculation of ligand-based similarity using Tanimoto coefficient and bit strings. As we can see, both known active compound and the database of untested compounds could be represented and further compared using molecular fingerprints, a high-dimensional sequence of bits that accounts for the absence (0) or presence (1) of representative fragments or atoms in each chemical structure (Cereto-Massagué et al., 2015; Gomes et al., 2017; Muegge and Mukherjee, 2016). Then, the comparison between bit vector sequences must be expressed using many similarity metrics. The most used metric to explore similarity is the Tanimoto coefficient, which is equal to the number of common bits set to 1 in both fingerprints divided by the total number of bits set to 1 between both fingerprints (Cereto-Massagué et al., 2015; Gomes et al., 2017; Muegge, Mukherjee, 2016). It assumes that two structures with Tanimoto coefficient higher than 0.85 can be considered structurally similar (Cereto-Massagué et al., 2015; Neves et al., 2016).

\section{Ligand-based pharmacophores and shape-based models}

Ligand-based pharmacophores and shape-based models aim to identify potentially active compounds based on their overlapping to the 3D arrangement of key interacting chemical features (e.g., aromatic rings, hydrogen bond donors or acceptors, partial charges, etc.) or shape and volume of known active ligands not shared by inactive compounds (Figure 1B) (Caporuscio, Tafi, 2011; Hawkins, Skillmann, Nicholls, 2007; Vuorinen, Schuster, 2015). In the same way, 3D shape-based models identify potentially active compounds based on their overlapping to the $3 \mathrm{D}$ surface shape of active compounds (Figure 1B) (Hawkins, Skillman, Nicholls, 2007; Koes, Camacho, 2014; Kortagere, Krasowski, Ekins, 2009). The generation of both models basically involves four steps: (i) alignment of a training set of molecules (composed by actives and inactives) into a known bioactive query; (ii) scoring of compounds and selection of best pharmacophoric/shape hypothesis; (iii) statistical validation by using appropriate metrics to determine the ability of the hypothesis to discriminate between known active and inactive compounds (Braga, Andrade, 2013); and (iv) screening of untested compounds using the statistically validated models.

\section{Quantitative Structure-Activity Relationships (QSAR)}

One of the most popular approaches for computeraided drug design is the quantitative structure-activity 
relationships (QSAR). QSAR is a method to quantify the correlation between the chemical structures of a set of compounds and a particular biological property (Gomes et al., 2017; Melo-Filho et al., 2016; Neves et al., 2016). QSAR modeling could be presented as three-part process (Figure 1C). Initially, chemical structures are converted into molecular descriptors (independent variables), which are the result of a logic and mathematical procedure that transforms chemical information into a useful number (Todeschini, Consonni, 2008). Then, machine learning methods (e.g., Random Forest (Breiman, 2001), Deep Learning (LeCun, Bengio, Hinton, 2015), Support Vector Machine (Vapnik, 2000 ), etc.) are used to establish quantitative relationships between descriptors and biological property (dependent variable). This step involves empirically discovering a function that will establish weights to molecular descriptors adjusting the equation $\mathrm{Bp}=\mathrm{k}^{\prime}\left(\mathrm{D}_{1}, \mathrm{D}_{2}, \ldots, \mathrm{Dn}\right)$, where $\mathrm{Bp}$ is biological property of molecules, $\mathrm{D}_{1}, \mathrm{D}_{2}, \ldots, \mathrm{Dn}$ are molecular descriptors, and k' is some empirically established weight assigned by the selected algorithm (Cherkasov et al., 2014; Tropsha, 2010). Lastly, the quality of the resulting QSAR model is measured by using appropriate metrics, which will check its ability to correctly predict the biological properties of experimentally evaluated compounds. Once validated, the generated model can be used in VS campaigns to prioritize untested compounds for synthesis and/or biological evaluation (Cherkasov et al., 2014; Tropsha, 2010).
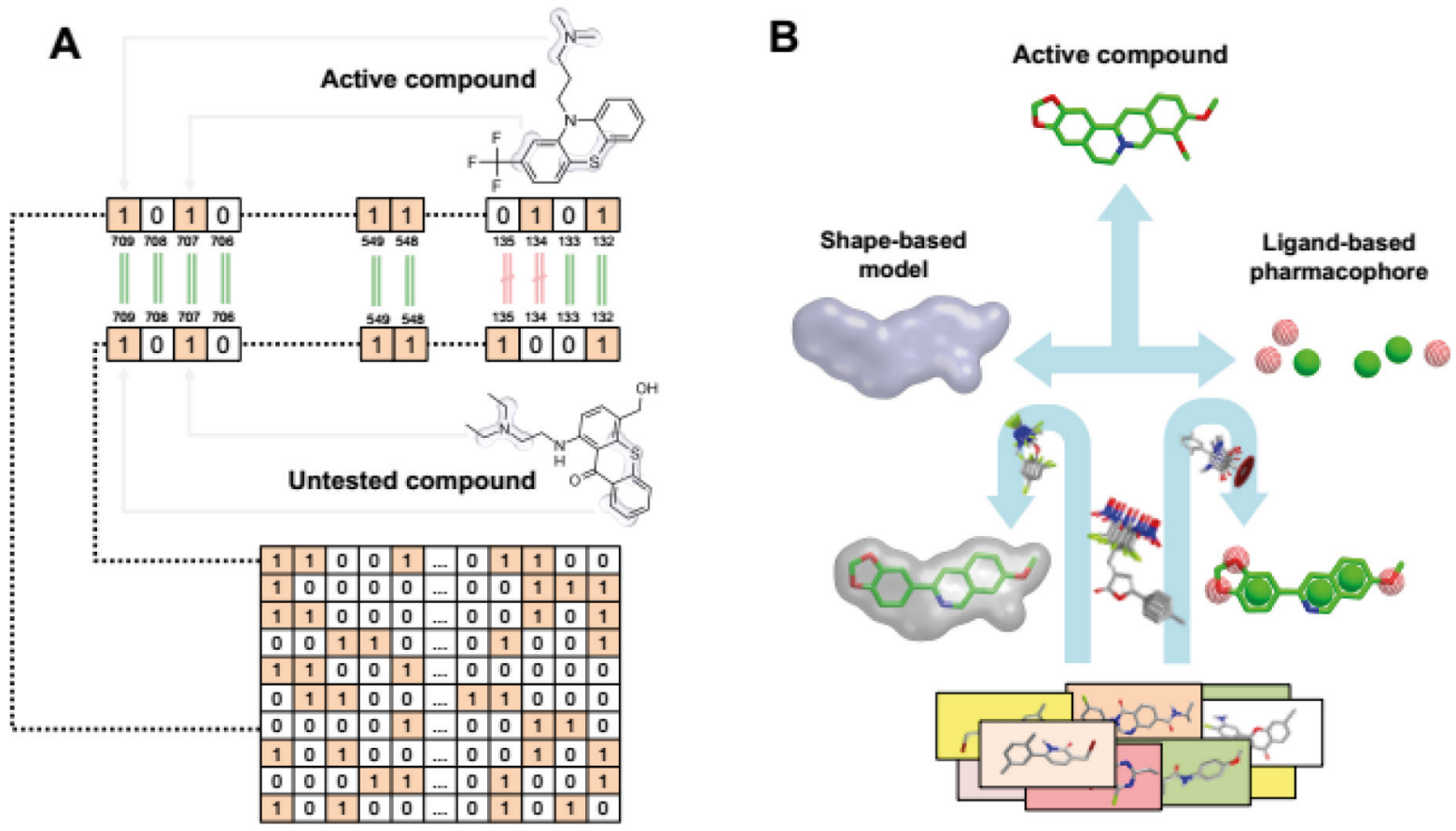

C
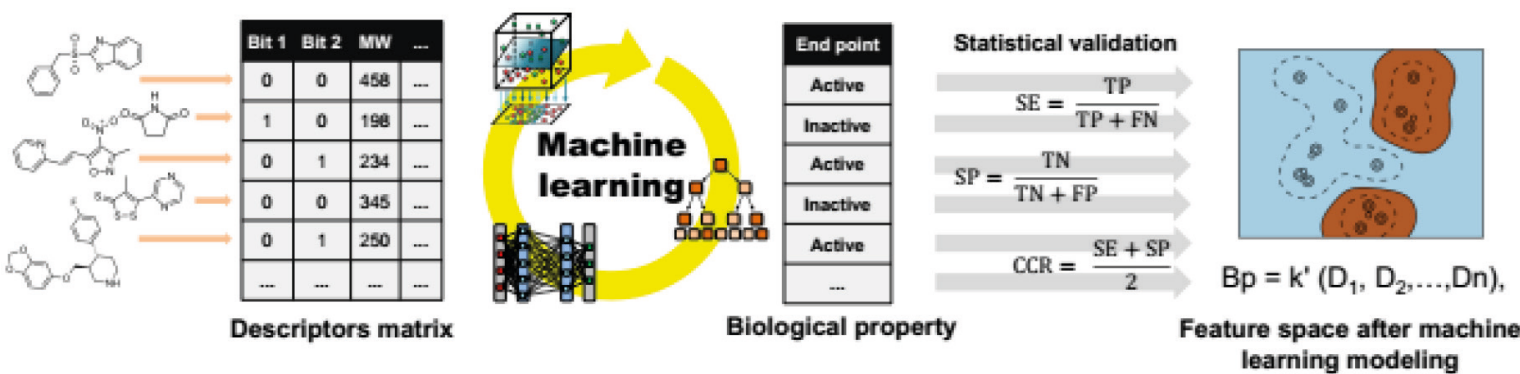

FIGURE 1 - Schematic representation of LBDD methods. (A) calculation of chemical similarity using bit strings (encoded by the presence and absence of structural fragments) generated for a known active compound and a database of untested chemicals; (B) fitting of a compound into pharmacophore and shape-based models generated from a known active ligand. Hydrogen bond acceptors are represented as red spheres, respectively and aromatic rings by green spheres; and (C) QSAR modeling using machine learning approaches and multiple statistical metrics. 
ZIKV bioassays, such as cell viability (in human astrocyte, astrocytoma and neural progenitor cells) and quantitative High-Throughput Screening (qHTS), related to caspase-3 activity, in human astrocyte, astrocytoma and iPSC derived neural progenitor cells, have been performed and are available in PubChem, sourced by NCATS Chemical Genomics Center (NCGC). Therefore, from these bioassays, QSAR modeling could be utilized as a filter in Virtual Screening of hits against ZIKV.

\section{Structure-based drug design (SBDD)}

Structure-based drug design (SBDD) is a very useful approach when the biological target is known, and its 3D structure is available. Based on the target 3D structural information, SBDD methods can assist the selection of ligands with good complementarity and affinity to the protein binding site (Lionta et al., 2014). In early march 2016, when the ZIKV outbreak started, there were no crystal structures for ZIKV proteins available and therefore there were several efforts to develop homology models based on close homologs such as DENV and others (Ekins et al., 2016a). Currently, 49 entries containing 3D structures of ZIKV proteins are available in Protein Data Bank (PDB) (Berman et al., 2000). Based on that, SBDD strategies represents a promising tool for assisting with the discovery of new drugs against ZIKV. In general, SBDD methods can be divided in three main categories: (i) protein-ligand docking; (ii) structure-based pharmacophores; and (iii) molecular dynamics. These approaches are discussed below.

\section{Protein-ligand docking}

Molecular docking is one of the most widely used SBDD methods, and largely applied in structure-based VS (SBVS) campaigns (Figure 2B). It consists of the computational fitting of ligands into the protein binding site, aiming to predict the ligand-protein complex, and estimating the ligand binding affinity (Ferreira et al., 2015). In general, the docking process is executed in two main steps: (i) the exploration of the conformational space, by generating various poses (orientations) of the ligand, which is executed by the search algorithm; and (ii) the use of scoring functions to perform the ranking of the most promising ligand poses, and estimate the binding affinity (Kalyaanamoorthy, Chen, 2011). There is a variety of search methods for exploring the conformational space of ligands, such as systematic methods (conformational search, databases), random or stochastic methods (Monte Carlo, genetic algorithms), and simulation methods (energy minimization, molecular dynamics). The scoring functions are generally divided in three groups: force field-based, empirical, and knowledge-based functions (Kalyaanamoorthy, Chen, 2011; Kitchen et al., 2004; Lionta et al., 2014).

\section{Structure-based pharmacophores}

Structure-based pharmacophore modeling takes advantage of the information about the 3D structure of proteins, and uses this information to probe interaction points between the protein and the ligand (Figure 2A) (Yang, 2010). There are two possibilities of calculating a SBP from a 3D protein structure: (i) The protein structure is complexed with a ligand (halo structure); and (ii) The protein has no complexed ligand (apo structure) (Pirhadi, Shiri, Ghasemi, 2013). In the first approach, the ligand binding site is identified, the key ligand-protein interactions are determined, and the gathered information is used to define the pharmacophore model (Yang, 2010). The second approach is based on the use of molecular probes (small fragments, functional groups) to map hot spots or possible interaction sites in the protein. These hot spots can be used as features, which are used to generate the pharmacophore model (Caporuscio, Tafi, 2011). The SBP approach can be used as a complement to docking studies, since it includes the same level of information. Furthermore, it demands less computational cost in comparison to docking, and represent a good alternative for researchers with limited computational resources (Braga, Andrade, 2013).

\section{Molecular dynamics simulations}

Molecular dynamics (MD) simulations provide detailed information about the behavior of a system and its temporal evolution on a molecular scale (Salsbury, 2010). Through this technique, it's possible to determine the motion of proteins and molecules, at atomic level, and the distinct interactions among them. The potential energy of interaction among atoms bonded (mainly including bond stretching, angle bending and dihedrals energies) or nonbonded (including van der Waals and electrostatic energies) is used to determine the atom systems dynamic (Figure 2C). From the coordinates, initial position and initial velocities of the atoms, force and acceleration are calculated following Newton's equations in order to obtain a temporal trajectory.

MD simulations have become valuable tool in probing details of biomolecules dynamics. This approach allows sampling different protein conformations, exploring the energy landscapes of proteins, and understanding protein functions. MD is also relevant in drug discovery and development, specially used to study the effects of ligand binding on protein dynamics (Amato et al., 2012), and the protein conformations associated with specific cellular function to enable the discovery of 
conformationally-selective ligands (Bernardes et al., 2013; Childers, Daggett, 2017; Mottin et al., 2017). Moreover, MD is useful in the structural refinements of post-docking complexes and in the analysis of protein-ligand complexes stability (G. Ricci et al., 2016; Musyoka et al., 2016; Ramharack, Soliman, 2018).

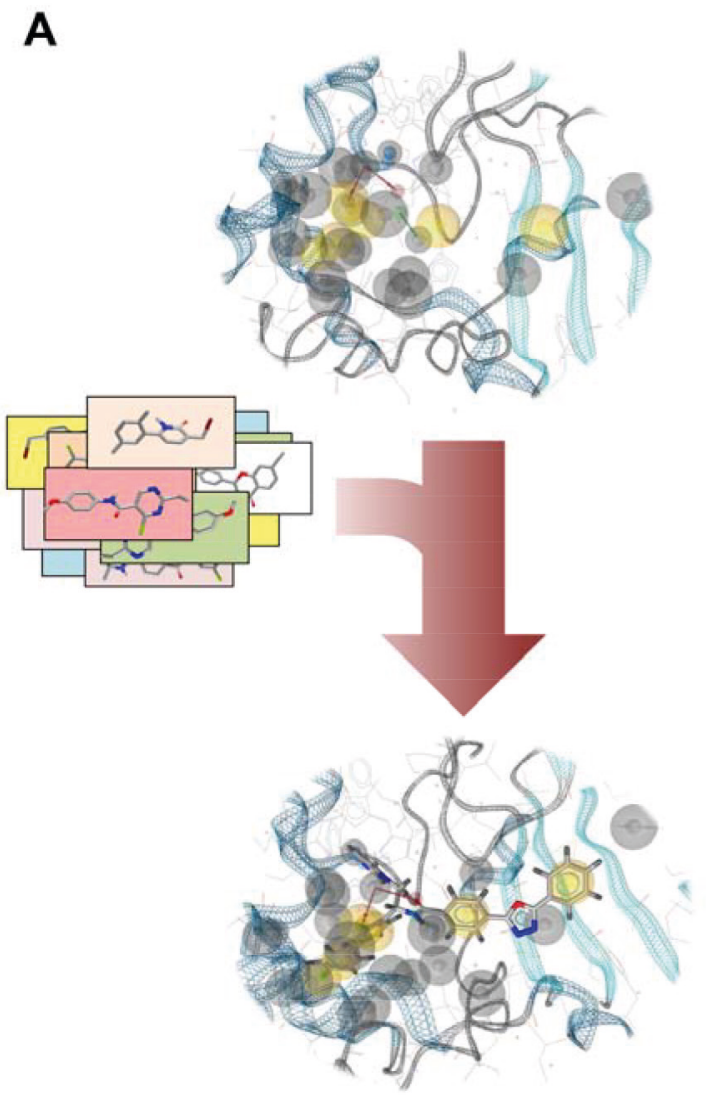

\section{In silico drug repurposing}

The main aim of drug repurposing (or drug repositioning) is to identify new uses for already existing drugs (Baker et al., 2018; Ekins et al., 2011). This approach accelerates the drug discovery process,

B
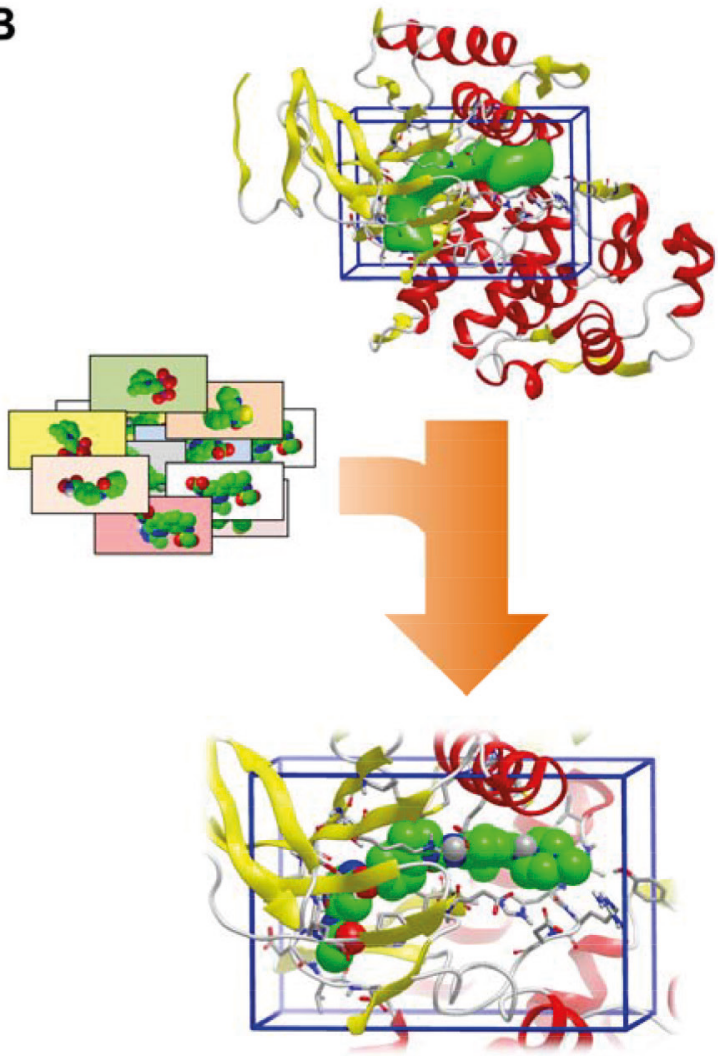
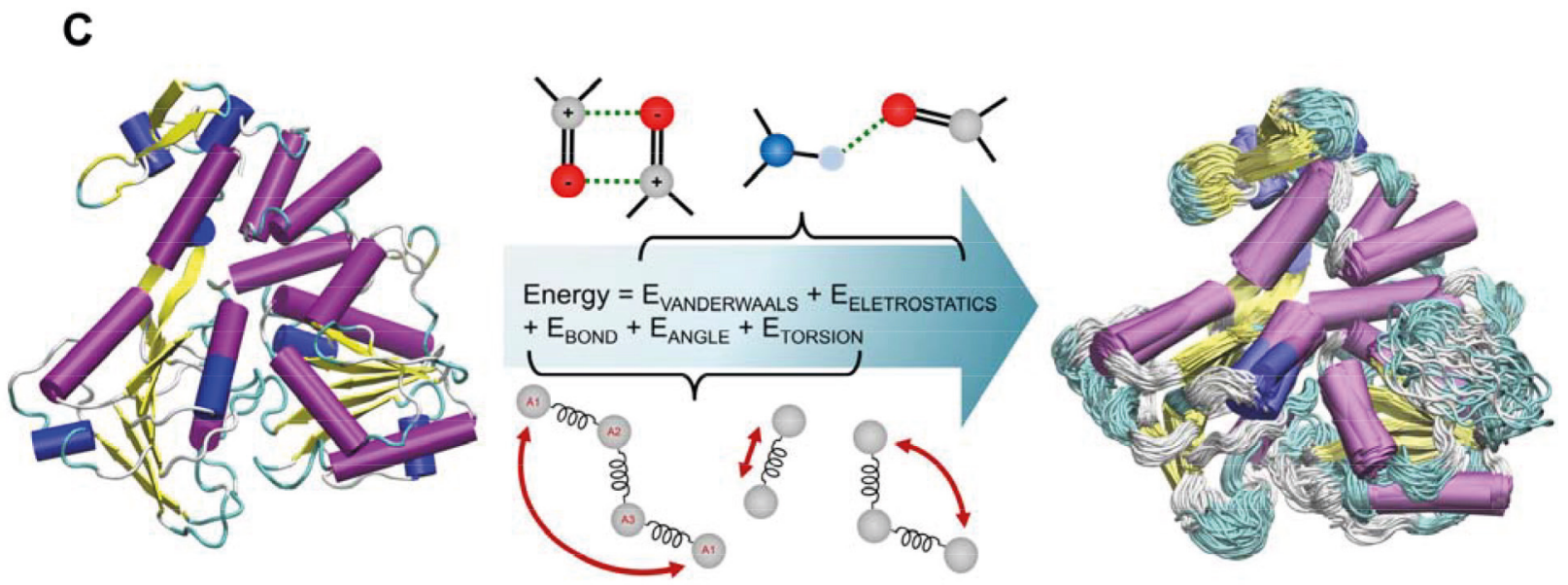

FIGURE 2 - Schematic representation of SBDD methods. (A) Structure-based pharmacophore model applied for VS of a large database of untested compounds. Yellow spheres represent hydrophobic features, red arrows are hydrogen bond acceptors, green arrow is a hydrogen bond donor feature, blue cone represents a metal interaction, and gray spheres are the exclusion volumes; (B) Ligand-protein docking applied for VS of a large database of untested compounds; and (C) Application of molecular dynamics simulations for insights of protein conformational changes, and the design of new anti-ZIKV drugs. 
reducing time efforts and expenses, also circumvents preclinical development (March-vila et al., 2017). Recent drug repurposing experimental studies (Barrows et al., 2016; Xu et al., 2016) demonstrated that screening approved or investigational drugs from datasets as FDA approved drugs, Library of Pharmacologically Active Compounds and NCATS pharmaceutical collection, is a promising strategy for identifying therapeutics with novel antiviral activity against ZIKV. Through this approach the following compounds have already been discovered as anti-ZIKV agents: niclosamide (Xu et al., 2016), PHA-690509 (Xu et al., 2016), emricasan (Xu et al., 2016), seliciclib (Xu et al., 2016), bortezomib (Barrows et al., 2016), mycophenolic acid (Adcock et al., 2017; Barrows et al., 2016), auranofin (Barrows et al., 2016), ivermectin (Barrows et al., 2016) and daptomycin (Barrows et al., 2016). Today, only experimental HTS screening were performed for ZIKV drug repurposing, but in silico methods are also a promising approach for ZIKV drug repurposing and have been successfully applied in other examples (March-vila et al., 2017). This represents an opportunity that has been under-utilized for ZIKV.

\section{In silico fragment-based drug discovery (FBDD)}

Fragment-Based Drug Discovery (FBDD) is a powerful computational technique to identify starting hits (small molecules or fragments) and then expand or link together to subsequent generate leads compounds (Mortier et al., 2012; Murray, Blundell, 2010; Zauhar, Gianti, Welsh, 2013). There are several strategies to perform fragment-based in silico approach, such as docking of fragment libraries; reapplication of de novo design programs to existing fragment collections; bioisosteric fragment replacement and pharmacophore screening for fragments (Mortier et al., 2012).

Since its development, a number of practical successful applications of FBDD have been reported in the literature for different targets (Mortier et al., 2012). Recently it has been used for DENV NS2B/NS3 protease (Frimayanti et al., 2012) and NS5 methyltransferase (Benmansour et al., 2017) proteins and represents a promising strategy for identifying hits for ZIKV.

\section{SUCCESSFUL APPLICATIONS COMPUTA- TIONAL APPROACHES FOR ZIKV DRUG DISCOVERY}

Virtual screening based on molecular docking has been used to select FDA-approved drugs, natural products and synthetic compounds for ZIKV non-structural proteins. The main focus of these VS are proteins involved in the viral replication complex process: NS3 helicase (Byler, Ogungbe, Setzer, 2016; Sahoo et al., 2016), NS5 RNA polymerase (Byler, Ogungbe, Setzer, 2016; Ramharack, Soliman, 2017) and NS5 methyltransferase (Byler, Ogungbe, Setzer, 2016; Ramharack, Soliman, 2017). NS2B/NS3 protease is also an important target due to its role in preprocessing the viral polyprotein (Byler, Ogungbe, Setzer, 2016; Lee et al., 2016; Sahoo et al., 2016). However, most of the published VS works do not validate their predictions with experimental assays, apart from one (Lee et al., 2016), which is a fundamental step to identifying new drug candidates.

One successful application of VS for ZIKV drug discovery has been performed in a collaboration with IBM World Community Grid (WCG) and some Universities/ Companies from Brazil and United States of America. This initiative, called the OpenZika project, is a collaborative open science project that virtually screens millions of compounds against all the ZIKV protein structures, through molecular docking and QSAR modelling (Figure 3 ). The OpenZika main goal is to accelerate the drug discovery for ZIKV, creating a net of volunteers computers that help scientific calculations (Ekins et al., 2016c). These results are publicly available, helping other researchers to focus on the most likely compounds that may eventually lead to a ZIKV antiviral. The docking calculations have been made for the main ZIKV proteins NS1, NS2B-NS3 protease, NS3 helicase and NS5 polymerase. From the beginning of the project (May 2016) until January 2018, $\sim 4.5$ billion dockings have been submitted in WCG and $\sim 3$ billion results have been received. Many of the virtually selected compounds have been selected for experimental validation.

The first published result of this project is related to ZIKV NS3 helicase, which is the main component of the replication complex of the virus. Helicase displays nucleoside 5'-triphosphatase (NTPase) and 5'-terminal RNA triphosphatase (RTPase) activities. The dynamic behavior of ZIKV helicase was firstly studied through molecular dynamics (MD) simulations by the OpenZika team (Mottin et al., 2017). This approach showed that a region near the RNA binding site, known as RNA binding loop, is influenced by the presence of the RNA strand, being more stable in this case. The analysis of different enzyme conformations, through the AutoLigand program (Forli et al., 2016; Harris, Olson, Goodsell, 2008), showed a region beneath/behind the ATP site that perhaps represents an allosteric site, and may could help the design of a more specific inhibitor for ATP site. 


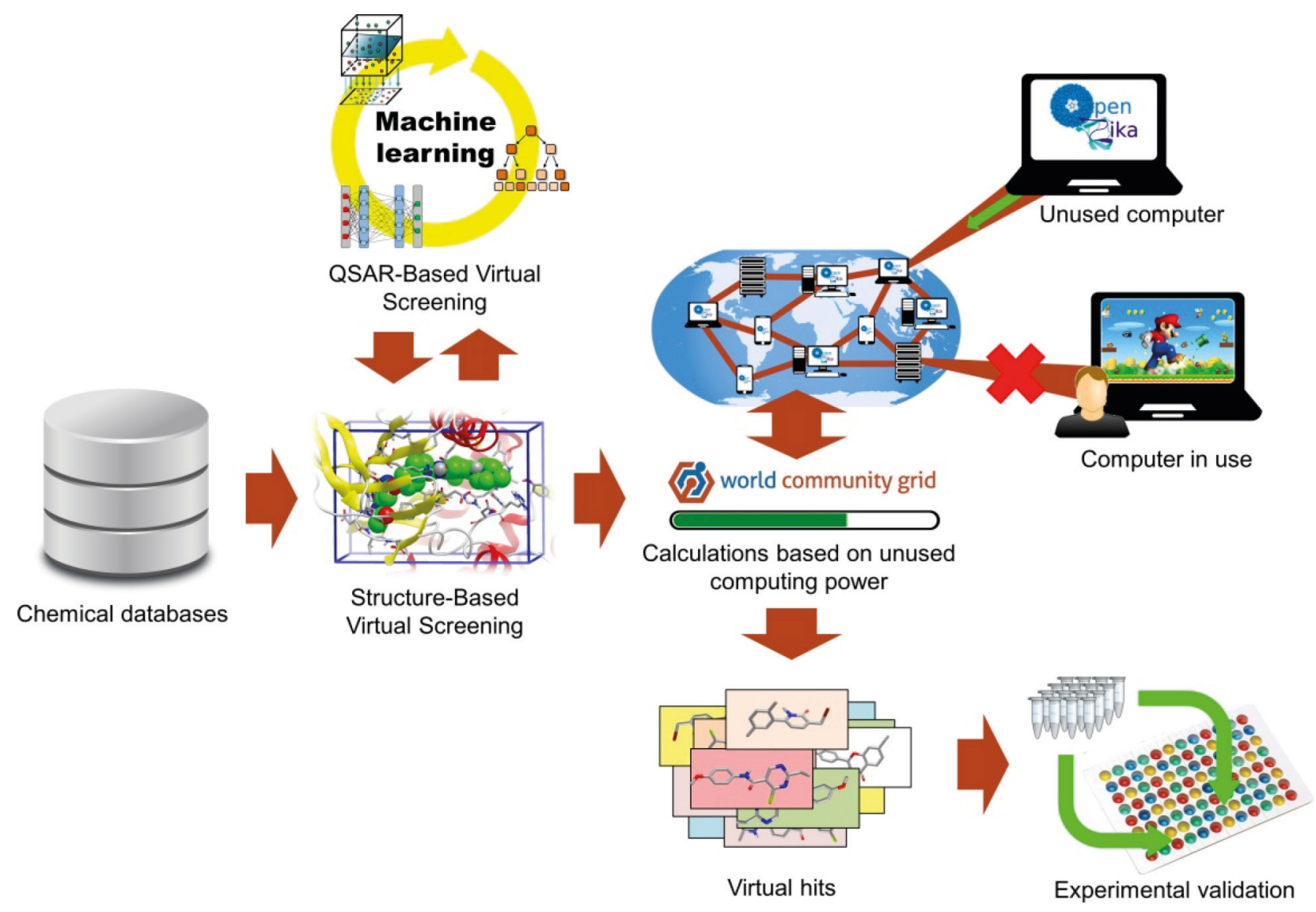

FIGURE 3 - Workflow of the OpenZika project: From Structure-Based Virtual Screening using molecular docking and QSAR modelling, millions of chemical compounds (from ZINC database) have been virtually screened against ZIKV protein structures. The calculations have been spread among volunteer's computers (using the idle computing power), provided by IBM's World Community Grid (WCG). All the selected virtual hits have been experimentally tested.

\section{SUMMARY}

ZIKV virulence and its devastating consequences to humans requires that we quickly discover new antivirals to stop this virus. This brief review highlights the computational technologies that are readily available to academia and industry, including both LBDD and SBDD. These have the potential to accelerate the process of drug discovery for ZIKV. We emphasize that, parallel to the computational strategies, it is important to perform the experimental validation and that there is a considerable opportunity for computational drug repurposing.

\section{ACKNOWLEDGEMENTS}

The authors would like to thank Brazilian funding agencies, CNPq, CAPES, FAPEG and FAPESP for grants and fellowships. This work was partially supported by the CNPq/FAPEG DCR (Grant 300508/2017-4). EM thanks the financial support from NIH (grant 1U01CA207160) and CNPq (grant 400760/2014-2). CHA thanks the FAPESP Visiting Researcher Program (grant 2017/023539). CHA is Research Fellow in productivity of CNPq. We kindly acknowledge all our collaborators on the OpenZika project (http://openzika.ufg.br), the wonderful ongoing support and encouragement of the IBM World Community Grid team, and the community of volunteers who donate their computer time.

\section{REFERENCES}

Adcock RS, Chu Y, Golden JE, Chung D. Evaluation of anti-Zika virus activities of broad-spectrum antivirals and NIH clinical collection compounds using a cell-based, highthroughput screen assay. Antiviral Res. 2017;138:47-56.

Amato AA, Rajagopalan S, Lin JZ, Carvalho BM, Figueira ACM, $\mathrm{Lu}$ J, et al. GQ-16, a novel peroxisome proliferator-activated receptor $\gamma(\operatorname{PPAR} \gamma)$ ligand, promotes insulin sensitization without weight gain. J Biol Chem. 2012;287(33):28169-79. 
Araújo TVB, Rodrigues LC, de Alencar Ximenes RA, de Barros Miranda-Filho D, Montarroyos UR, de Melo APL, et al. Association between Zika virus infection and microcephaly in Brazil, January to May, 2016: preliminary report of a casecontrol study. Lancet Infect Dis. 2016;16(12):1356-63.

Baker NC, Ekins S, Williams AJ, Tropsha A. A bibliometric review of drug repurposing. Drug Discov Today. 2018;23(3):661672.

Barrows NJ, Campos RK, Powell ST, Routh A, Bradrick SS, Garcia-blanco MA, et al. A screen of FDA-approved drugs for inhibitors of Zika virus infection resource a screen of FDAapproved drugs for inhibitors of Zika virus infection. Cell Host Microbe. 2016;20(2):259-70.

Benmansour F, Trist I, Coutard B, Decroly E, Querat G, Brancale A, et al. Discovery of novel dengue virus NS5 methyltransferase non-nucleoside inhibitors by fragment-based drug design. Eur J Med Chem. 2017;125:865-80.

Berman HM, Westbrook J, Feng Z, Gilliland G, Bhat TN, Weissig H, et al. The protein data bank. Nucleic Acids Res 2000;28(1):235-42.

Bernardes A, Souza PCT, Muniz JRC, Ricci CG, Ayers SD, Parekh NM, et al. Molecular mechanism of peroxisome proliferator-activated receptor $\alpha$ activation by WY14643: a new mode of ligand recognition and receptor stabilization. J Mol Biol. 2013;425:(16)2878-2893.

Braga RC, Andrade CH. Assessing the performance of 3D pharmacophore models in virtual screening: how good are they? Curr Top Med Chem. 2013;13(9):1127-38.

Breiman L. Random forests. Machine Learning. 2001;45(1):532.

Byler KG, Ogungbe IV, Setzer WN. In-silico screening for antiZika virus phytochemicals. J Mol Graph Model. 2016;69:78-91.

Caporuscio F, Tafi A. Pharmacophore modelling: a forty year old approach and its modern synergies. Curr Med Chem. 2011;18(17):2543-53.

Cereto-Massagué A, Ojeda MJ, Valls C, Mulero M, GarciaVallvé S, Pujadas G. Molecular fingerprint similarity search in virtual screening. Methods. 2015;71:58-63.
Cherkasov A, Muratov EN, Fourches D, Varnek A, Baskin II, Cronin M, et al. QSAR modeling: Where have you been? Where are you going to? J Med Chem. 2014;57(12):4977-5010.

Childers MC, Daggett V. Insights from molecular dynamics simulations for computational protein design. Mol Syst Des Eng. 2017;2(1):9-33.

Dick GWA, Kitchen SF, Haddow AJ. Zika virus. I. Isolations and serological specificity. Trans R Soc Trop Med Hyg. 1952;46(5):509-20.

Duffy MR, Chen T-H, Hancock WT, Powers AM, Kool JL, Lanciotti RS, et al. Zika Virus Outbreak on Yap Island, Federated States of Micronesia. N Engl J Med. 2009;360:2536-43.

Ekins S, Liebler J, Neves BJ, Lewis WG, Coffee M, Bienstock $\mathrm{R}$, et al. Illustrating and homology modeling the proteins of the Zika virus. F1000Res. 2016a;5:275.

Ekins S, Mietchen D, Coffee M, Stratton T, Freundlich J, Freitas-Junior L, et al. Open drug discovery for the Zika virus. F1000Res. 2016b;5:1-12.

Ekins S, Perryman AL, Horta Andrade C. OpenZika: An IBM World Community Grid Project to Accelerate Zika Virus Drug Discovery. PLoS Negl Trop Dis. 2016c;10(10):e0005023.

Ekins S, Williams AJ, Krasowski MD, Freundlich JS. In silico repositioning of approved drugs for rare and neglected diseases. Drug Discov Today. 2011;16(7-8):298-310.

Ferreira L, dos Santos R, Oliva G, Andricopulo A. Molecular docking and structure-based drug design strategies. Molecules. 2015;20(7):13384-421.

Forli S, Huey R, Pique ME, Sanner MF, Goodsell DS, Olson AJ. Computational protein - ligand docking and virtual drug screening with the AutoDock suite. Nat Protoc. 2016;11(5):90519.

Frimayanti N, Zain SM, Sanghiran V, Wahab HA. Fragmentbased molecular design of new competitive dengue Den2 Ns2b / Ns3 inhibitors from the components of fingerroot (Boesenbergia rotunda). In Silico Biol. 2012;11(1-2):29-37.

G. Ricci C, Liu Y-L, Zhang Y, Wang Y, Zhu W, Oldfield E, et al. Dynamic structure and inhibition of a malaria drug target: geranylgeranyl diphosphate synthase. Biochemistry. 2016;55(36):5180-90. 
Glaab E. Building a virtual ligand screening pipeline using free software: a survey. Brief Bioinform. 2016;17(2):352-66.

Gomes MN, Muratov EN, Pereira M, Peixoto JC, Rosseto LP, Cravo PVL, et al. Chalcone derivatives: promising starting points for drug design. Molecules. 2017;22(8):E1210.

Gulland A. Zika virus is a global public health emergency, declares WHO. BMJ 2016:1657.

Harris R, Olson AJ, Goodsell DS. Automated prediction of ligand-binding sites in proteins. Proteins Struct Funct Bioinforma. 2008;70(4):1506-17.

Hawkins PCD, Skillman AG, Nicholls A. Comparison of shapematching and docking as virtual screening tools. J Med Chem 2007;50(1):74-82.

Hennessey M, Fischer M, Staples JE. Virus spreads to new areas - Region of the Americas.CDC. 2016;65(3):55-58.

Joy Macalino SY, Vijayakumar Gosu B, Sunhye Hong B, Sun Choi B. Role of computer-aided drug design in modern drug discovery. Arch Pharm Res. 2015;38(9):1686-701.

Kalyaanamoorthy S, Chen Y-PP. Structure-based drug design to augment hit discovery. Drug Discov Today. 2011;16(1718):831-9.

Kar S, Roy K. How far can virtual screening take us in drug discovery? Expert Opin Drug Discov. 2013;8(3):245-61.

Kitchen DB, Decornez H, Furr JR, Bajorath J. Docking and scoring in virtual screening for drug discovery: methods and applications. Nat Rev Drug Discov. 2004;3(11):935-49.

Koes DR, Camacho CJ. Shape-based virtual screening with volumetric aligned molecular shapes. J Comput Chem. 2014;35(25):1824-34.

Kortagere S, Krasowski MD, Ekins S. The importance of discerning shape in molecular pharmacology. Trends Pharmacol Sci. 2009;30(3):138-47.

LeCun Y, Bengio Y, Hinton G. Deep learning. Nature. 2015;521:436-44.

Lee H, Ren J, Nocadello S, Rice AJ, Ojeda I, Light S, et al. Identification of novel small molecule inhibitors against NS2B/NS3 serine protease from Zika virus. Antiviral Res. 2016;139:49-58.
Lionta E, Spyrou G, Vassilatis DK, Cournia Z. Structure-based virtual screening for drug discovery: principles, applications and recent advances. Curr Top Med Chem 2014;14(16):1923-38.

March-vila E, Pinzi L, Sturm N, Tinivella A, Engkvist O, Chen $\mathrm{H}$, et al. On the integration of in silico drug design methods for drug repurposing. Front Pharmacol. 2017;8:1-7.

Melo-Filho CC, Dantas RF, Braga RC, Neves BJ, Senger MR, Valente WCG, et al. QSAR-driven discovery of novel chemical scaffolds active against schistosoma mansoni. J Chem Inf Model. 2016;56(7):1357-72.

Mortier J, Rakers C, Frederick R, Wolber G. Computational tools for in silico fragment-based drug design. Curr Top Med Chem. 2012;12(17):1935-43.

Mottin M, Braga RC, da Silva RA, Martins da Silva JH, Perryman AL, Ekins S, et al. Molecular dynamics simulations of Zika virus NS3 helicase: Insights into RNA binding site activity. Biochem Biophys Res Commun. 2017;492(4):643-651.

Muegge I, Mukherjee P. An overview of molecular fingerprint similarity search in virtual screening. Expert Opin Drug Discov. 2016;11(2):137-48.

Murray CW, Blundell TL. Structural biology in fragment-based drug design. Curr Opin Struct Biol. 2010;20(4):497-507.

Musyoka TM, Kanzi AM, Lobb KA, Tastan Bishop Ö. Structure based docking and molecular dynamic studies of plasmodial cysteine proteases against a South African natural compound and its analogs. Sci Rep. 2016;6:23690.

Neves BJ, Dantas RF, Senger MR, Melo-Filho CC, Valente WCG, de Almeida ACM, et al. Discovery of new anti-schistosomal hits by integration of QSAR-based virtual screening and high content screening. J Med Chem. 2016;59(15):7075-88.

Neves BJ, Muratov E, Machado RB, Andrade CH, Cravo PVL. Modern approaches to accelerate discovery of new antischistosomal drugs. Expert Opin Drug Discov. 2016;11(6):557-67.

Oehler E, Watrin L, Larre P, Lastère S, Valour F, Baudouin L, et al. Zika virus infection complicated by Guillain-Barré syndrome - case report, French Polynesia, December 2013. Euro Surveil. 2014;19(9):pii20720. 
Pirhadi S, Shiri F, Ghasemi JB. Methods and applications of structure based pharmacophores in drug discovery. Curr Top Med Chem. 2013;13(9):1036-47.

Ramharack P, Soliman MES. Zika virus NS5 protein potential inhibitors: an enhanced in silico approach in drug discovery. J Biomol Struct Dyn. 2018;36(5):1118-1133.

Reynolds CH. Impact of computational structure-based methods on drug discovery. Curr Pharm Des. 2014;20(20):3380-3306.

Ripphausen P, Nisius B, Bajorath J. State-of-the-art in ligandbased virtual screening. Drug Discov Today. 2011;16(9-10):3726.

Sahoo M, Jena L, Daf S, Kumar S. Virtual screening for potential inhibitors of NS3 protein of Zika Virus. Genomics Inform. 2016;14(3):104-11.

Salsbury FR. Molecular dynamics simulations of protein dynamics and their relevance to drug discovery. Curr Opin Pharmacol. 2010;10(6):738-44.

Tanrikulu Y, Krüger B, Proschak E. The holistic integration of virtual screening in drug discovery. Drug Discov Today. 2013;18(7-8):358-64.

Todeschini R, Consonni V. Handbook of molecular descriptors. Weinheim, Germany: Wiley-VCH Verlag GmbH; 2008. http:// doi.wiley.com/10.1002/9783527613106

Tropsha A. Best practices for QSAR Model development, validation, and exploitation. Mol Inform. 2010;29(6-7):476-88.
Vapnik V. The Nature of Statistical Learning Theory. 2nd ed. New York: Springer; 2000.

Ventura C V, Maia M, Bravo-Filho V, Góis AL, Belfort R. Zika virus in Brazil and macular atrophy in a child with microcephaly. Lancet. 2016;387(10015):228.

Vuorinen A, Schuster D. Methods for generating and applying pharmacophore models as virtual screening filters and for bioactivity profiling. Methods. 2015;71:113-34.

Wahid B, Ali A, Rafique S, Idrees M. Zika: As an emergent epidemic. Asian Pac J Trop Med. 2016;9(8):723-9.

Xu M, Lee EM, Wen Z, Cheng Y, Huang W, Qian X, et al. Identification of small-molecule inhibitors of Zika virus infection and induced neural cell death via a drug repurposing screen. Nat Med. 2016;22(10):1101-1107.

Yang S-Y. Pharmacophore modeling and applications in drug discovery: challenges and recent advances. Drug Discov Today. 2010;15(11-12):444-50.

Zauhar RJ, Gianti E, Welsh WJ. Fragment-based Shape Signatures: a new tool for virtual screening and drug discovery. J Comput Aided Mol Des. 2013;27(12):1009-1036.

Zhu T, Cao S, Su P-C, Patel R, Shah D, Chokshi HB, et al. Hit identification and optimization in virtual screening: practical recommendations based on a critical literature analysis. J Med Chem. 2013;56(17):6560-72. 